\section{Inclusão da variável tempo em sistemas de informação geográfica}

Resumo: Incorporar o elemento temporal em Sistemas de informação geográfica (SIG) é um desafio que vem sendo pesquisado por muitos anos e apresenta diversas propostas de solução. Para a inserção da variável tempo em SIG, diversas questões devem ser levadas em consideração. O presente artigo aborda algumas dessas questões. Inicialmente apresentam-se os estágios no desenvolvimento de SIG espaçotemporais, em seguida, discute-se a questão da representação de dados em SIG e depois são apresentados os conceitos relacionados à dimensão temporal no contexto de SIG. A semântica dos dados espaço-temporais também é abordada, seguida pela definição das funções a que um SIG com características espaço-temporais deve atender e a abordagem da questão do suporte a consultas. Por fim, apresentam-se alguns dos principais modelos desenvolvidos para a representação de dados espaçotemporais em SIG.

\section{Including time in geographic information systems}

\begin{abstract}
To incorporate the temporal element in traditional geographic information systems (GIS) is a challenge that has been researched for many years and has had several proposed solutions. In order to insert time in GIS, several issues must be taken into consideration, and this study addresses some of them. Firstly, we present the stages in the development of space-time GIS. Then, we discuss the issue of data representation in GIS and present the concepts related to the temporal dimension in the context of GIS. Next, the semantics of spatio-temporal data is discussed. We then define the functions that a GIS with spatial and temporal characteristics must meet and address the issue of supporting queries. Finally, we present some of the main models developed for the representation of spatio-temporal GIS.
\end{abstract}

\section{Alexandro Gularte Schafer* Ruth Emilia Nogueira**}

*Professor MSc. da UNIPAMPA. Engenheiro Civil, mestre em Engenharia Civil. Doutorando em Engenharia Civil área de Cadastro Técnico e Gestão Territorial.

** Professora Dra. do departamento de Geociências da UFSC. Coordenadora do programa de pós-graduação em Geografia da UFSC.

Palavras-chave: Sistemas de informação geográfica; Tempo; Representação temporal

Key-words: Geographic information systems; Time; Temporal representation 


\section{Introdução}

Os Sistemas de Informação Geográfica (SIG) são projetados para capturar, armazenar, visualizar, comunicar, transformar e analisar informação geo-referenciada (GOODCHILD, 2008). Embora os SIG constituam uma tecnologia capacitada para abordar problemas que envolvam 0 aspecto temporal dos dados, segundo Peuquet (2002), a questão de como representar a dinâmica espaço-temporal nesses sistemas foi ignorada até o final dos anos 1980. De fato, diversos autores, entre eles Hornsby e Egenhofer (2000), Worboys (2001); McMaster e Usery (2004); Worboys e Hornsby (2004); Ahola et al. (2007), argumentam que, ainda hoje, a maioria dos SIG são estáticos.

De acordo com Yuan (1996), um SIG com características temporais tem como objetivo processar, gerenciar e analisar dados espaço-temporais. Esses sistemas melhoram os SIG existentes (estáticos ou atemporais), pois proporcionam a manipulação da componente temporal do dado. Segundo Abraham e Roddick (1999), a possibilidade de considerar cenários passados, presente e futuros do ambiente modelado no sistema proporciona novas características aos SIG, superando a capacidade dos SIG estáticos atuais.

Segundo Couclelis (2005), a complexidade da gerência, da manutenção e da operação dos aspectos espaço-temporais gera, atualmente, uma forte demanda de pesquisa nesta área, já que muitas questões desafiadoras ainda permanecem sem solução. Segundo Le (2005), a modelagem de dados espaço-temporal e o desenvolvimento de sistemas de informação geográfica temporal são elementos-chave na busca pela incorporação da variável tempo em Sistemas de informação geográfica. Neste sentido, o presente artigo tem como objetivo abordar algumas dessas questões trazendo os estágios no desenvolvimento deste tipo de SIG, representação de dados, os conceitos envolvidos na dimensão temporal em SIG, a semântica relativa aos dados e as funções a que um SIG com características espaço-temporais deve atender. Também apresentam-se alguns dos principais modelos desenvolvidos para a representação de dados espaço-temporais em SIG.

\section{Estágios no desenvolvimento de sistemas de informação geográfica espaço-temporais}

Worboys (2005) descreve quatro estágios principais na introdução da dimensão temporal em sistemas de informação geográfica. São eles o estágio zero (SIG estático), o estágio um (instantâneos temporais), o estágio dois (mudança do objeto) e o estágio três (eventos, ações e processos).

O estágio zero é, em geral, onde a tecnologia SIG comercial está atualmente. Neste estágio é possivel representar o passado ou o futuro, mas somente um único momento no tempo pode ser representado. Além disso, não é possível fazer comparações entre os estados do objeto entre diferentes datas.

No estágio um, o mundo é visualizado como uma sucessão de configurações espaciais de objetos em instâncias temporais. Esta tem sido a abordagem mais comum em modelos espaçotemporais até agora. Um instantâneo temporal é uma representação do estado de um objeto de estudo em um domínio particular e em um único momento no tempo. Uma sequência de instantâneos é uma coleção de instantâneos temporais, geralmente todos da mesma área, indexados por uma variável temporal. Não é realmente o domínio do tempo que dita estas propriedades, mas a natureza do fenômeno que está sendo considerado. 
No estágio dois, o foco muda da sequência temporal de objetos, seus atributos e relacionamentos, para mudanças que podem acontecer a objetos, a atributos e a relacionamentos. Um modelo do mundo baseado na evolução de objetos ao longo do tempo, retendo identidade, mas modificando atributos espaciais e outros, parece natural. Entretanto, surgem problemas relacionados à continuidade da identidade do objeto ao longo do tempo.

O estágio três na evolução dos sistemas de informação espaço-temporal é um tratamento completo de mudança em termos de eventos, ações e processos. No estágio três espera-se que sejam modelados eventos complexos, a maneira com que objetos podem participar nesses eventos e as relações entre os eventos.

\section{Representação de dados em sistemas de informações geográficas}

De acordo com Yuan et al. (2004), a representação geográfica constitui-se em um elemento central no desenvolvimento de um SIG. Ela é uma maneira de comunicar a informação geográfica e é também uma estrutura binária em um meio computacional ou de armazenamento eletrônico que corresponde a um objeto, medida ou fenômeno do mundo. A representação em SIG inclui três aspectos das entidades do mundo real: sua localização na superfície da terra, seus atributos e qualquer relacionamento importante entre entidades (GOODCHILD, 2005). Como a representação proporciona fundamentos conceituais e computacionais para processar, integrar, analisar e visualizar dados geográficos, a representação escolhida para um fenômeno geográfico tem um profundo impacto em sua interpretação e análise (YUAN, 2010).

Os sistemas de informação geográfica e as análises espaciais são influenciados pelas representações em três níveis distintos: o nível de modelo de dados, o nível de formalização e 0 nível de visualização. 0 modelo de dados é o cerne conceitual de um sistema de informação, ele define as entidades, os relacionamentos, as operações e as regras para manter a integridade do banco de dados (CODD, 1980 in YUAN et al., 2004).

Peuquet (1984) define modelo de dados como uma descrição geral de conjuntos específicos de entidades e das relações entre estes conjuntos de entidades. Segundo 0 autor, a característica mais básica de um modelo de dados é que ele é uma abstração da realidade. Como os SIG não são capazes de processar a informação que está além das capacidades de representação de seus modelos de dados, a representação geográfica e os modelos de dados são críticos para melhorar 0 processamento de consultas e as análises da informação geográfica (WORBOYS et al., 1990).

Nas quatro últimas décadas, testemunha-se uma massiva proliferação de modelos de dados SIG, estrutura de dados e discussões envolvendo representação e ontologia geográfica. A matriz geográfica de Berry (BERRY, 1964) e o esquema tridimensional de Sinton (SINTON, 1978) proporcionaram antigos modelos do mundo geográfico, como representações vetoriais e matriciais. Nos anos 1970, as estruturas de dados topológicas foram extensivamente discutidas; nos anos 1980, verificou-se que o modelo relacional oferecia uma maneira simples de implementar estruturas topológicas em SIGs comerciais (WORBOYS, 1995). Nos anos 1990, 0 modelo orientado a objeto foi amplamente adotado, embora frequentemente utilizando tecnologia de banco de dados relacional. Recentemente, esforços são realizados no sentido de estender estes modelos para incluir a dimensão temporal (GOODCHILD et al., 2007). Nesse processo de desenvolvimento dos SIGs, a distinção entre os conceitos de objeto discreto e campo contínuo recebe considerável atenção.

Geografia Ensino \& Pesquisa, v. 16, n.1, p. 155172, jan.jun. 2012

Schafer, A. G.; Nogueira, R. E. 
Couclelis (2005) argumenta que a distinção entre a representação com base em campo e a com base em objeto foi fundamental para 0 entendimento do mundo em nossa volta, e fortemente relacionado à percepção humana. Segundo Goodchild et al. (2007), os conceitos de objeto discreto e campo continuo que foram introduzidos no final dos anos 1980 e inicio dos anos 1990, têm dominado o pensamento sobre a concepção humana do espaço geográfico.

$\mathrm{Na}$ visão de campo, a realidade é modelada por variáveis que possuem uma distribuição contínua no espaço. Toda posição no espaço geográfico pode ser caracterizada através de um conjunto de atributos medidos para um conjunto de coordenadas geográficas. Na prática, a variação contínua dos atributos é percebida como um conjunto de elementos discretos. 0 conceito de modelo baseado em campo origina-se da física clássica e tem sido usado para modelagem de propriedades físicas (e não-físicas), onde a magnitude é dependente de sua localização espacial (KJENSTAD, 2006).

$\mathrm{Na}$ visão de objeto, a realidade consiste de entidades individuais, bem definidas e identificáveis. Cada entidade tem suas propriedades e ocupa um determinado lugar no espaço. $A$ realidade é modelada como um grande espaço onde entidades estão distribuídas sem que, necessariamente, todas as posições do espaço estejam ocupadas. Duas ou mais entidades podem estar situadas sobre uma mesma posição geográfica. 0 modelo baseado em objeto tem sido usado como um meio de estruturação conceitual da informação geográfica, em particular na modelagem de objetos do mundo real (ou entidades) com localização e extensão espacial precisas (KJENSTAD, 2006).

Outros conceitos são o de objeto campo e o de campo de objetos. Yuan (1999) define objeto campo como um geo-objeto com heterogeneidade interna semelhante a um campo. Cova e Goodchild (2002) apresentam o conceito de campo de objetos, no qual cada ponto no mapa não é um valor, mas um geo-objeto.

Goodchild et al. (2007) introduzem os conceitos de geo-átomo e mostram que ele pode proporcionar a base tanto para a concepção de objeto discreto quanto de campo contínuo. Como os geo-campos e geo-objetos são os únicos elementos disponíveis na teoria proposta, pode-se inferir que objetos discretos e campos contínuos são de fato as únicas bases possíveis para a concepção do mundo geográfico. A teoria é limitada pelo seu foco na concepção baseada em conjuntos de pontos, e assim na agregação de geo-átomos em geo-campos e em geo-objetos.

De acordo com Peuquet (2002), a tradicional dicotomia campo-objeto utilizada na modelagem de SIG tem resultado em uma relativa negligência de representações de dados geográficos com base no tempo na comunidade de pesquisa. Esse terceiro elemento da perspectiva o que/onde/quando é necessário para representar explicitamente as situações de entidades e localizações e os seus inter-relacionamentos ao longo do tempo. Certamente, é possivel representar a informação temporal utilizando-se representações convencionais, mas isso acarreta em um significante aumento no volume de dados armazenados e na complexidade das operações.

Segundo Peuquet $(2001,2005)$, existem basicamente três abordagens para a representação dos dados espaço-temporais: representação com base em localização, representação com base em entidade e representação com base no tempo. Segundo a autora, é possível ainda desenvolver abordagens combinadas a partir dessas três. Partindo dessas abordagens, vários modelos de dados espaço-temporais vêm sendo propostos.

A incorporação do tempo em modelos que se baseiam em localização frequentemente é vista como uma solução óbvia para representação da mudança espacial. Tratar o tempo como espaço é algo útil para melhorar a percepção em contextos de problemas específicos. Entretanto, 
ele não permite que as propriedades únicas e especificas do tempo sejam explicitamente representadas (PEUQUET, 2002).

$\mathrm{Na}$ representação com base em entidade para dados espaço-temporais, os modelos propostos registram explicitamente mudanças espaciais ao longo do tempo relacionadas a entidades geográficas específicas ao invés de localizações. Em um nível conceitual, todos os modelos propostos com essa abordagem representam extensões da abordagem vetor topológica. Como tais, eles localizam mudanças na geometria de entidades ao invés do tempo. Um caso particular da abordagem com base em entidade é a abordagem orientada a objeto. (PEUQUET, 2005).

$\mathrm{Na}$ abordagem que tem como base o tempo, a localização no tempo se torna a base organizacional primária para o registro de mudanças. A sequência de eventos ao longo do tempo, representando a manifestação espaço-temporal de algum processo, é percebida via linha do tempo ou vetor temporal (uma linha unidimensional na dimensão tempo ao invés de uma superfície bi-dimensional sobre o espaço). Com este tipo de representação, as mudanças relativas ao tempo são explicitamente armazenadas (PEUQUET, 2005).

\section{A dimensão temporal em SIG}

O tempo é um elemento essencial para fins de localização e análise visando 0 estabelecimento de padrões, predição de eventos futuros ou explicação de ocorrências. A informação temporal pode ter diferentes formas quando dados geográficos são coletados. Pode ser um simples instante de tempo, ou pode ser uma expressão de duração. Dados geográficos podem ser coletados a intervalos regulares, ou podem ser registrados somente quando uma mudança ocorre. Em se tratando de SIG, os tipos de tempo auxiliam na decisão de como analisar o que acontece em determinados locais, determinar quando ocorrem mudanças e no examine de vários cenários possíveis (VASILIEV, 1997).

Muitos conceitos fundamentais sobre tempo e propriedades do tempo têm sido profundamente discutidos na literatura da ciência da computação e da informação geográfica. Progressos significantes vêm sendo alcançados no desenvolvimento conceitual assim como em estudos de caso em diferentes domínios de aplicação (YUAN, 2008).

Conforme Worboys e Dunckham (2004), o tempo pode ser representado por meio de diferentes estruturas, definidas principalmente com base em três elementos da representação temporal: Variação temporal (contínua ou discreta); ordem no tempo (linear, ramificado e circular) e granularidade (instante de tempo, intervalo de tempo, período ou elemento temporal). A figura 01 apresenta as estruturas temporais segundo Worboys e Dunckham (2004).

Quanto à variação temporal, considera-se a possibilidade de variação contínua e variação discreta. A variação temporal contínua é usada em processos que demandam medidas de tempo com níveis arbitrários de precisão. Já a variação temporal discreta é usada quando o tempo é medido em certos pontos ou intervalos de tempo, e a variação é descontínua entre estes pontos (WORBOYS e DUCKHAM, 2004). Um intervalo de tempo é o tempo entre dois instantes. Um instante pode ser definido como um ponto no tempo em um eixo temporal.

Geografia Ensino \& Pesquisa, v. 16, n.1, p. 155172, jan.jun. 2012

Schafer, A. G.; Nogueira, R. E.

ISSN 2236- 4994 


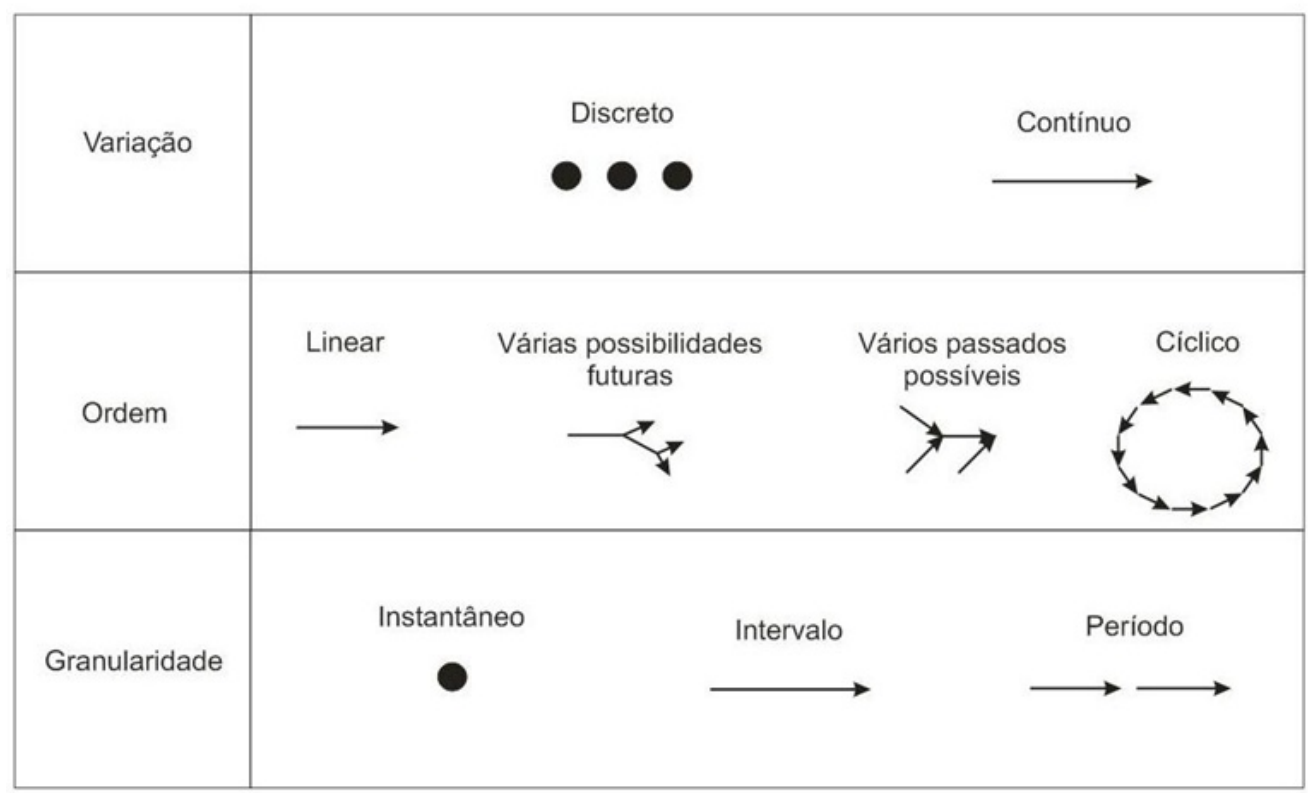

Figura 01- Estruturas temporais (modificado de Worboys e Duckham, 2004)).

Embora a modelagem de processos dinâmicos ou mudanças contínuas seja identificada como objetivo de pesquisa (YUAN et al., 2004), grande parte da literatura na comunidade SIG trata as mudanças espaço-temporais de maneira discreta, o que é relativamente mais fácil (HORNSBY e EGENHOFER, 2000).

Com relação à ordem, o tempo pode ser classificado como linear (ou linearmente ordenado), circular (ou cíclico) ou ramificado (WORBOYS e DUNCKHAM, 2004). 0 tempo linear considera que os pontos da linha do tempo fluem sequencialmente (há uma ordem de precedência entre eles) e os eventos podem ser medidos em escala ordinal ou em intervalos. 0 tempo circular representa tempos recorrentes e combina ordem de eventos e seus intervalos de tempo de ocorrência. Neste caso, a periodicidade de sua ocorrência faz com que sempre se volte a mesma referência de tempo (DIAS et al., 2005). 0 tempo ramificado permite que dois pontos diferentes sejam sucessores (ramificação no futuro) ou antecessores (ramificação no passado) imediatos de um mesmo ponto. Para ambos a restrição linear é abandonada.

Embora o espaço e o tempo sejam contínuos, eles são convencionalmente divididos em unidades discretas, de comprimento uniforme ou variável para propósitos de medição objetiva. Associado à variação temporal discreta está o conceito de granularidade. Intuitivamente, a granularidade define uma partição, possivelmente não total, do domínio do tempo (CAMOSSI et al., 2006). Associado a essa variação, existe o conceito de chronon, que pode ser definido como a menor duração de tempo suportada por um sistema, podendo variar em diferentes aplicações (EDELWEISS e OLIVEIRA, 1994). Qualquer atividade em um banco de dados temporal acontece durante o período de pelo menos um chronon. Os elementos primitivos de representação da granularidade temporal são o instante de tempo (representa um ponto particular de tempo), o intervalo de tempo (tempo decorrido entre dois pontos) e o período ou elemento temporal (sequência de intervalos de tempo) (WORBOYS e DUCKHAN, 2004).

Do ponto de vista do armazenamento em banco de dados, de acordo com Snodgrass (1992) existem dois tipos de tempo, o tempo de validade e o tempo de transação. O tempo de validade é o momento em que o evento ocorreu. $O$ tempo de transação é o momento em que essa ocorrência foi registrada no banco de dados. Conforme a presença ou ausência do registro 
do tempo de validade e/ou do tempo de transação, quatro tipos de Sistemas Gerenciadores de Banco de Dados (SGBDpodem ser identificados: estático, de tempo de validade (histórico), de tempo de transação (rollback) e bitemporal. Um SGBD estático não mantém nem o tempo de validade nem o tempo de transação. Um SGBD histórico lida somente com o tempo de validade, podendo recuperar o histórico de um evento de acordo com o tempo de validade registrado. Um SGBD de tempo de transação registra o instante da inserção de dados no $\mathrm{BD}$, possibilitando uma recuperação de dados para desfazer uma transação. Um SGBD bitemporal registra tanto 0 tempo de validade quanto o tempo de transação (WORBOYS, 1994). 0 quadro 01 (SNODGRASS, 1992; WORBOYS, 1994; WORBOYS e DUCKHAN, 2004; DIAS et al., 2005) indica a dimensão temporal mantida em cada caso.

\begin{tabular}{|c|c|c|}
\hline & Sem tempo de transação & Com tempo de transação \\
\hline Sem tempo de validade & Estático & Por tempo de transação (rollback) \\
\hline Com tempo de validade & Histórico (por tempo de validade) & bitemporal \\
\hline
\end{tabular}

Quadro 01- Classificação dos Sistemas Gerenciadores de Banco de Dados segundo a dimensão temporal

\section{Semântica dos dados espaço-temporais}

Existem, em particular, duas grandes categorias de conceitos geográficos com as quais as pessoas estão familiarizadas: entidades e fenômenos geográficos (e as propriedades e relações espaciais e temporais que as caracterizam). Os fenômenos geográficos são abstratos ou concretos e ocorrem na natureza de forma contínua ou discreta. Os fenômenos geográficos são distintos de dados geográficos, os quais são feições selecionadas (geralmente numéricas) que os geógrafos usam para descrever ou medir direta ou indiretamente o fenômeno que tem a qualidade de ser espacial. Por exemplo, o fenômeno clima pode ser visto em parte através dos dados da precipitação (DENT, 1996 in LOCH, 2006). Os fenômenos são analisados através de dados que podem ser qualitativos ou quantitativos e que podem ser descritos nominalmente, ordinalmente, ou proporcionalmente, e representados por pontos, áreas, linhas, usando variáveis visuais tais como cor, tamanho, forma, etc. As entidades podem ser consideradas como os elementos ou classes representados em meio analógico ou digital. A segunda categoria de conceitos geográficos universalmente compartilhados refere-se a noções de espaço e tempo aplicáveis a escalas geográficas, e em particular as relações espaciais e temporais entre entidades e fenômenos geográficos (COUCLELIS, 2005).

A visão de temporalidade compartilhada pela maioria dos pesquisadores relaciona-se a uma sequência de estados pontuados por eventos que transformam um estado no próximo (LANGRAN, 1992). Um elemento central na modelagem espaço-temporal são as mudanças que ocorrem ao longo do tempo e a maneira de representá-la em SIG (AHOLA et al., 2007).

A mudança é normalmente descrita como um evento ou coleção de eventos. Cada mutação de um objeto é um evento que produz uma nova versão do objeto e um novo estado do mapa (LANGRAN, 1992). Para propósitos de modelagem espaço-temporal, Peuquet (2005) define evento como "uma mudança no estado de uma ou mais localizações, entidades ou ambos". Os eventos são necessários para capturar os mecanismos de mudança (WORBOYS e HORNSBY, 2004).

Peerbocus et al. (2004) citam três tipos principais de mudança de documentação no contexto de gerenciamento de dados espaço-temporais, com base em três perspectivas no

Geografia Ensino \& Pesquisa, v. 16, n.1, p. 155172, jan.jun. 2012

Schafer, A. G.; Nogueira, R. E.

ISSN 2236- 4994 
contexto da representação espaço-temporal em SIG (Figura 02): a) documentação sobre eventos ocorrendo no mundo real, constituindo o aspecto semântico da evolução; b) documentação referente a evolução cartográfica, descrevendo as diferentes modificações que o usuário faz no nivel de interface e, c) documentação sobre a evolução do banco de dados, contendo informações sobre os diferentes objetos que estão sendo atualizados.

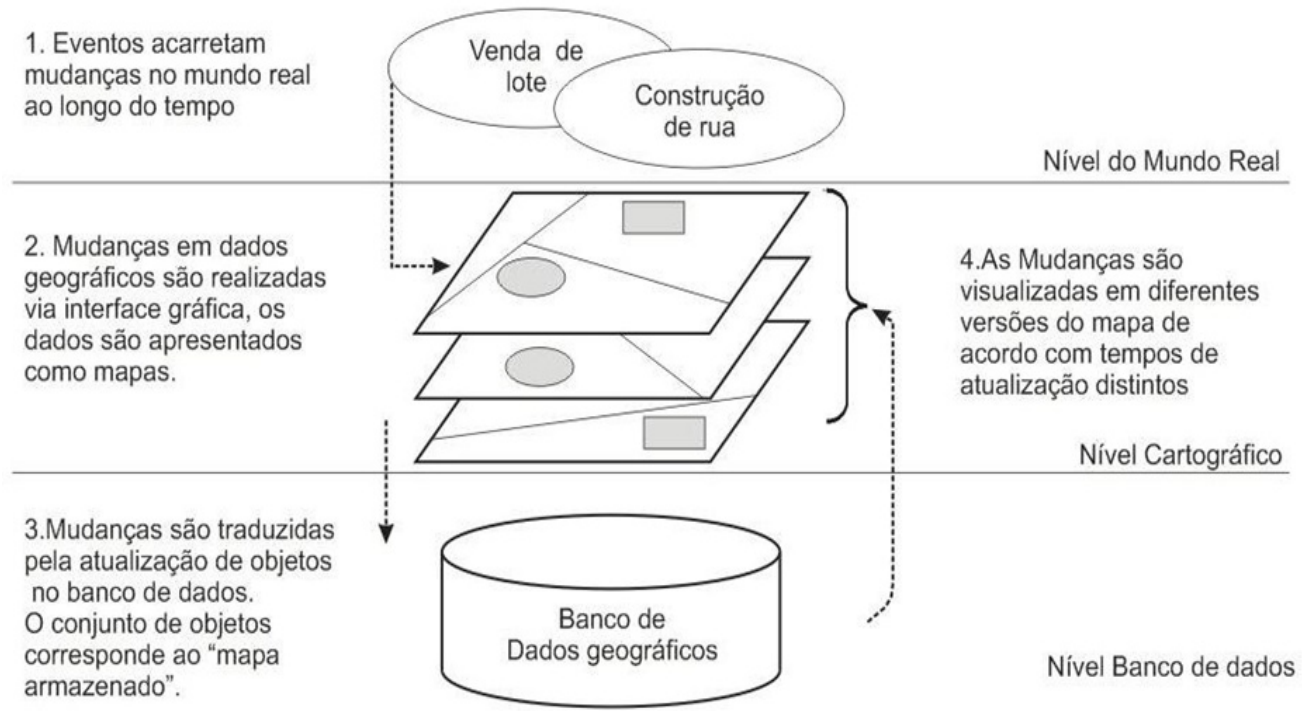

Figura 02-Perspectivas no contexto da representação espaço-temporal em SIG e diferentes níveis de mudança (modificado de Peerbocus et al., 2004).

Do ponto de vista do mundo real, uma mudança refere-se a um evento do mundo real, que é uma consequência de fenômenos naturais ou da ação humana. De um ponto de vista do sistema de informação (ou perspectiva cartográfica), Peerbocus et al. (2004) consideram que 0 nível cartográfico é a interface do usuário com o banco de dados espaço-temporal. 0 usuário visualiza os dados armazenados no BD através de mapas. Como a perspectiva cartográfica corresponde a interface com o sistema, é através deste nível que as mudanças no mundo real se refletem no banco de dados.

No nível do banco de dados, um evento do mundo real é representado por atualizações isto é, criação, destruição e modificação - dos objetos no banco de dados. A granularidade das mudanças pode variar de um nível para o outro. No nível do banco de dados, cada atualização corresponde a uma mudança, considerando que no nível cartográfico - que é o nível que o usuário visualiza e com o qual ele interage - uma mudança pode corresponder a várias operações de atualização de dados (PEERBOCUS et al., 2004).

Sinton (1978) e Yuan (1996a) investigaram diferentes tipos de mudanças espaço-temporais e como elas podem ser medidas, representadas e analisadas em SIG. Em se tratando de objetos, Armstrong (1988) argumenta que oito subtipos de mudanças temporais resultam da combinação de mudanças em seus atributos, morfologia e topologia (figura 03).

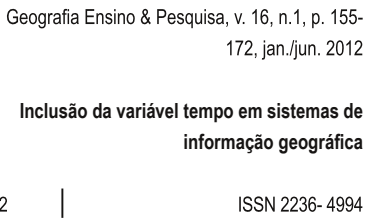




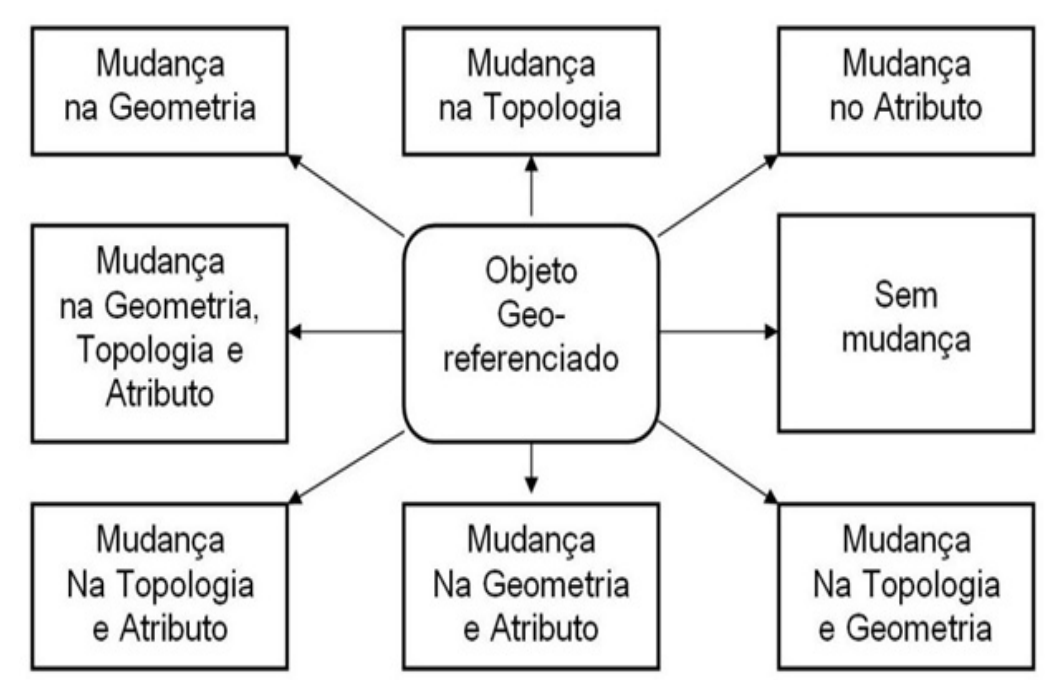

Figura 03- Oito possíveis mudanças espaço-temporais de um objeto geográfico (modificado de Sinton, 1978).

Goodchild et al. (2007) argumentam que a dinâmica do comportamento de objetos pode ser capturada em três dimensões fundamentais, caracterizadas como estrutura interna, geometria e movimento.

$\mathrm{Na}$ dimensão relacionada à geometria do objeto, distingue-se entre objetos que permanecem com a mesma forma e objetos que mudam de forma ao longo do tempo. $\mathrm{Na}$ dimensão relacionada à estrutura interna do objeto, distingue-se entre objetos que são homogêneos e objetos que tem variação interna. Na dimensão relacionada ao movimento, distingue-se entre objetos que se movem e aqueles que permanecem fixos em um local ao longo do tempo.

Com relação aos tipos de dados, tendo por base as características espaciais, semânticas e temporais, Price et al. (1999) definem cinco tipos diferentes de dados para aplicações espaçotemporais: dados espaciais, dados temporais, dados espaço-temporais, dados semânticos e dados compostos (quadro 02).

\begin{tabular}{|c|c|}
\hline Tipo de dado & Característica \\
\hline Dados espaciais & possuem somente domínio espacial \\
\hline Dados temporais & possuem somente domínio temporal \\
\hline Dados espaço-temporais & dados espaciais que se alteram com o tempo \\
\hline Dados semânticos & $\begin{array}{r}\text { podem ter variações em consequência de mudanças puramente } \\
\text { espaciais, temporais ou espaço-temporais }\end{array}$ \\
\hline Dados compostos & $\begin{array}{r}\text { constituídos por associação de dados e cujos componentes } \\
\text { podem mudar com o tempo ou espacialmente }\end{array}$ \\
\hline
\end{tabular}

Quadro 02-Tipos de dados para aplicações espaço-temporais.

\section{Funções de aplicações com características espaço-temporais}

Um SIG com características temporais tem como objetivo processar, gerenciar e analisar dados espaço-temporais (YUAN, 1996). Uma importante questão a ser considerada na construção de um SIG com características temporais são as funções a que esse SIG deve

Geografia Ensino \& Pesquisa, v. 16, n.1, p. 155172, jan./jun. 2012

Schafer, A. G.; Nogueira, R. E.

ISSN 2236- 4994 
atender. De acordo com Langran (1992), as funções de um SIG temporal são inventário, análise, atualização, controle de qualidade, visualização e agendamento (que é opcional). 0 quadro 03 apresenta essas funções.

\begin{tabular}{|c|c|}
\hline Função & Descrição \\
\hline Inventário & $\begin{array}{l}\text { Armazena uma descrição completa da área de estudo e considera as } \\
\text { mudanças no mundo físico e no meio computacional. }\end{array}$ \\
\hline Análise & $\begin{array}{l}\text { Explica, explora ou prevê os componentes e os processos atuando em uma } \\
\text { determinada área. }\end{array}$ \\
\hline Atualização & Atualiza informação desatualizada por informação corrente. \\
\hline Controle de qualidade & $\begin{array}{l}\text { Avalia onde novos dados apresentam inconsistência lógica, de acordo com } \\
\qquad \text { versões e estados prévios. }\end{array}$ \\
\hline Agendamento & $\begin{array}{l}\text { Identifica ou antecipa estados limiares do banco de dados que ativam } \\
\text { respostas pré-definidas do sistema. }\end{array}$ \\
\hline Visualização & $\begin{array}{c}\text { Gera um mapa estático ou dinâmico, ou uma tabela, de processos temporais } \\
\text { atuantes em uma determinada área. }\end{array}$ \\
\hline
\end{tabular}

Quadro 03 - Principais funções de um SIG temporal.

\section{Suporte a consultas}

Segundo Yuan e Mclntosh (2002), o suporte a consultas é uma das funções mais importantes de um sistema de informação. A identificação dos tipos de consultas tem profunda implicação no projeto de um sistema de informação espaço-temporal e no processamento e mineração da informação espaço-temporal. As consultas refletem o tipo de informação que 0 usuário deseja obter. Um sistema de informação deve ter a habilidade de representar as entidades de interesse visando obter respostas acerca dessas entidades.

Yuan e Mclntosh (2002) tomaram como base os trabalhos de Langran (1992), Peuquet (1994), Yuan (1996) e Yuan (1999) para propor uma tipologia de consultas espaço-temporais (quadro 04) de acordo com os elementos de informação, buscando suporte ao processamento automático de consultas.

\begin{tabular}{|c|c|c|c|}
\hline \multicolumn{2}{|c|}{ Tipo de consulta } & Definição & Exemplo \\
\hline \multicolumn{2}{|c|}{ Atributo } & $\begin{array}{l}\text { Tem como base informação própria sobre um } \\
\text { objeto identificado ou uma localização em um } \\
\text { campo. Em muitos casos, tais consultas } \\
\text { podem ser respondidas mediante a } \\
\text { recuperação de dados gravados que cumprem } \\
\text { certo conjunto de critérios. Frequentemente } \\
\text { envolvem operação booleana. }\end{array}$ & $\begin{array}{c}\text { Que país tem densidade populacional } \\
\text { maior do que } 500 \text { habitantes por } \mathrm{km}^{2} \text { ? } \\
\text { Que medidor de vazão tem um } \\
\text { período de registro de dados maior do } \\
\text { que } 5 \text { anos? }\end{array}$ \\
\hline Espacial & Simples & $\begin{array}{l}\text { Tenta recuperar entidades espaciais sujeitas } \\
\text { a critérios definidos. Usualmente não envolve } \\
\text { computação geométrica, já que a seleção é } \\
\text { feita por recuperação de dados gravados que } \\
\text { satisfazem um dado conjunto de critérios. }\end{array}$ & $\begin{array}{l}\text { Onde estão os medidores } \\
\text { administrados pelo USGS? Onde o solo } \\
\text { é arenoso? }\end{array}$ \\
\hline
\end{tabular}




\begin{tabular}{|c|c|c|c|}
\hline \multirow[t]{2}{*}{ Espacial } & Série espacial & $\begin{array}{l}\text { Busca informação sobre atributos ou } \\
\text { entidades dentro de uma área específica. A } \\
\text { área pode ser definida por entidades } \\
\text { geográficas com limites determinados, ou pela } \\
\text { proximidade a uma entidade geográfica. A } \\
\text { consulta de série espacial requer computação } \\
\text { geométrica através de sobreposição espacial } \\
\text { para selecionar objetos espaciais que se } \\
\text { posicionam dentro de uma área definida. É } \\
\text { uma das mais populares consultas em SIG } \\
\text { para análise exploratória e planejamento. }\end{array}$ & $\begin{array}{l}\text { Quais tipos de uso e cobertura do solo } \\
\text { existem nesta bacia hidrográfica e qual é } \\
\text { a área de cada tipo existente? Qual é a } \\
\text { temperatura média anual no estado do } \\
\text { Rio Grande do Sul? }\end{array}$ \\
\hline & $\begin{array}{l}\text { Relacionamento } \\
\text { espacial }\end{array}$ & $\begin{array}{c}\text { Investiga como objetos e fenômenos se } \\
\text { relacionam no espaço. Os relacionamentos } \\
\text { espaciais podem ser classificados de acordo } \\
\text { com a proximidade e a topologia. Consultas de } \\
\text { proximidade espacial podem ser manipuladas } \\
\text { como consulta de série espacial quando a } \\
\text { proximidade definida é usada para fixar uma } \\
\text { série espacial (usualmente construindo buffers } \\
\text { em volta de um objeto específico). }\end{array}$ & $\begin{array}{l}\text { Quais estradas cruzam a ferrovia? } \\
\text { Quantas casas estão localizadas a } \\
\text { jusante deste rio? Qual é o caminho } \\
\text { mais curto (em distância ou tempo) do } \\
\text { local A para o local B? }\end{array}$ \\
\hline \multirow{3}{*}{$\begin{array}{l}\text { Temporal } \\
\text { (considerando } \\
\text { tempo linear e } \\
\text { tempo de } \\
\text { validade) }\end{array}$} & Simples & $\begin{array}{c}\text { Busca informação sobre o estado de uma } \\
\text { entidade em um dado momento (um } \\
\text { instantâneo). Distintamente da consulta a } \\
\text { atibutos, a consulta temporal simples pode ou } \\
\text { não ter registros correspondentes ao momento } \\
\text { investigado. }\end{array}$ & $\begin{array}{c}\text { Quando ocorreu a pior tempestade } \\
\text { neste mês? Quem foi o proprietário } \\
\text { desta casa em 1995? }\end{array}$ \\
\hline & Série temporal & $\begin{array}{c}\text { Questiona sobre o que acontece a uma } \\
\text { entidade ao longo de um determinado período } \\
\text { de tempo. A consulta de série temporal busca } \\
\text { informação sobre o desenvolvimento (ou o não } \\
\text { desenvolvimento) de um dado fenômeno no } \\
\text { espaço durante um período de tempo } \\
\text { especíico. }\end{array}$ & $\begin{array}{l}\text { Quão rápido esta tempestade se } \\
\text { moveu na última hora? Quantas vezes } \\
\text { este lote mudou de proprietário desde } \\
1950 ?\end{array}$ \\
\hline & $\begin{array}{l}\text { Relacionamento } \\
\text { temporal }\end{array}$ & $\begin{array}{c}\text { Questiona sobre como múltiplas entidades } \\
\text { se relacionam no tempo. Relacionamentos } \\
\text { temporais consistem de proximidade temporal } \\
\text { e topologia temporal. Proximidade temporal } \\
\text { considera entidades que ocorrem dentro de } \\
\text { um período de tempo com relação ao evento } \\
\text { em questão. A topologia temporal pode ter } \\
\text { como base pontos no tempo ou intervalos de } \\
\text { tempo. }\end{array}$ & $\begin{array}{l}\text { Quantos deslizamentos de terra } \\
\text { ocorreram em uma semana de } \\
\text { ocorrência dne tempestade na última } \\
\text { estação chuvosa? }\end{array}$ \\
\hline $\begin{array}{l}\text { Espaço- } \\
\text { temporal }\end{array}$ & Simples & $\begin{array}{l}\text { Questiona onde os objetos de interesse } \\
\text { existem em um dado momento, quando os } \\
\text { objetos de interesse existem em um dado local, } \\
\text { ou quando existem em um dado local em um } \\
\text { dado momento. Esta consulta pode envolver } \\
\text { somente dados consultados diretamente } \\
\text { através de seleção e operações booleanas. }\end{array}$ & $\begin{array}{l}\text { Onde João estava em 1997? Quando } \\
\text { estas dunas de areia se estenderam em } \\
\text { 100m a partir da linha costeira? }\end{array}$ \\
\hline
\end{tabular}

Geografia Ensino \& Pesquisa, v. 16, n.1, p. 155172, jan.jun. 2012

Schafer, A. G.; Nogueira, R. E. 


\begin{tabular}{|c|c|c|}
\hline $\begin{array}{l}\text { Série espaço- } \\
\text { temporal }\end{array}$ & $\begin{array}{l}\text { Questiona sobre o que acontece a uma } \\
\text { região ao longo de um período de tempo. } \\
\text { Mudanças somente podem ocorrer a partes } \\
\text { do fenômeno em questão ou causar diferenças } \\
\text { fundamentais nas propriedades da entidade. }\end{array}$ & $\begin{array}{c}\text { Que partes de uma área rural em } \\
\text { 1/1/1980 mudaram para área residencial } \\
\text { em 31/12/1989 em São Paulo? Onde } \\
\text { houve mudança na vegetação nesta } \\
\text { bacia hidrográfica entre 1/1/1980 e } \\
\text { 31/12/1989? }\end{array}$ \\
\hline $\begin{array}{l}\text { Comportamento } \\
\text { espaço-temporal }\end{array}$ & $\begin{array}{l}\text { Busca informação sobre mudança em } \\
\text { objetos, fenômenos, eventos ou processos no } \\
\text { espaço e no tempo, incluindo localização, } \\
\text { tamanho, forma, partes espaciais, frequência } \\
\text { de ocorrência, padrões de movimento e } \\
\text { distribuição de intensidade. O foco aqui é o } \\
\text { exame de atributos de entidades espaço- } \\
\text { temporais de um determinado tipo e como } \\
\text { estas propriedades se modificam a partir do } \\
\text { exame da percepção dos mecanismos que são } \\
\text { responsáveis pela mudança. Mudanças em } \\
\text { padrões de distribuição e estruturas no espaço } \\
\text { e tempo são também de interesse. }\end{array}$ & $\begin{array}{l}\text { Quanto a precipitação e a temperatura } \\
\text { variaram ao longo do curso desta } \\
\text { tempestade? Onde estão as áreas em } \\
\text { que houve aumento na probabilidade de } \\
\text { enchentes nos últimos } 10 \text { anos? }\end{array}$ \\
\hline $\begin{array}{l}\text { Relacionamento } \\
\text { espaço-temporal }\end{array}$ & $\begin{array}{c}\text { Questiona sobre como entidades de } \\
\text { diferentes tipos se relacionam umas com as } \\
\text { outras no espaço e no tempo. Teoricamente, } \\
\text { relacionamentos espaço-temporais podem ser } \\
\text { examinados de duas perspectivas: (1) } \\
\text { mudanças em relacionamentos espaciais ao } \\
\text { longo do tempo, e (2) mudanças em } \\
\text { relacionamentos temporais sobre o espaço. A } \\
\text { consulta de relacionamento espaço-temporal } \\
\text { busca a informação que é central para o } \\
\text { entendimento de eventos e processos e como } \\
\text { eles interagem na dinâmica do mundo real. }\end{array}$ & $\begin{array}{l}\text { Onde e quando é provável ocorrer a } \\
\text { precipitação de máxima intensidade } \\
\text { durante o desenvonlvimento de uma } \\
\text { tempestade? Onde e quando } \\
\text { inundações alcançarão a área } \\
\text { residencial a jusante, de acordo com a } \\
\text { propagação desta tempestade? }\end{array}$ \\
\hline
\end{tabular}

Quadro 04-Tipologia das consultas espaço-temporais

\section{Modelos para representação de dados espaço-temporais em SIG}

De acordo com Peuquet (2002), o desenvolvimento da capacidade temporal em modelos de dados geográficos, e SIG em geral, começou com o inovador trabalho de Langran no final dos anos 1980 (LANGRAN e CHRISMAN, 1988). A partir daí, vários modelos de dados espaçotemporais que incorporam tempo em SIG vêm sendo propostos. No quadro 05 apresentam-se, em ordem cronológica, alguns desses modelos. 


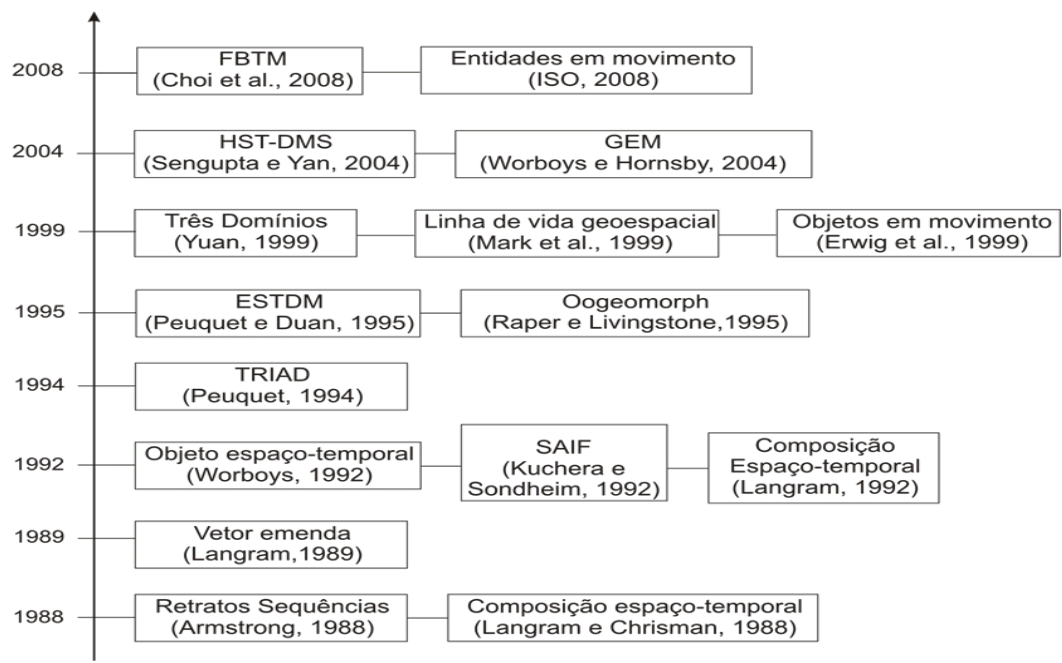

Quadro 05—Modelos para representação de dados espaço-temporais em SIG (organizado pelos autores).

- Em 1988, o modelo de Armstrong e o modelo de Langran e Chrisman foram a tentativa inicial de organizar conceitualmente dados espaço-temporais. Armstrong apresentou o modelo "Retratos Sequenciais" (Sequent Snapshots), que tem como base o tempo para organizar as mudanças ocorridas em uma localização. Langran e Chrisman propuseram o modelo Composição Espaço-Temporal (Space-Time Composite - STC), que se baseia em localização.

- Em 1989 Langran apresentou o modelo "estado inicial com emendas" (base state with amendments), que armazena somente as mudanças (isto é, emendas). Este modelo se baseia em entidades geográficas.

- Em 1992 Kuchera e Sondheim apresentaram o SAIF (Spatial Archive and Interchange Format) (Sondheim et al., 1997), orientado a objeto. Worboys propôs o modelo "Objeto Espaçotemporal" (Spatiotemporal Object Model), que tem como base objetos espaciais e Langran apresentou uma versão do modelo composição espaço-temporal baseado na abordagem campo.

- Em 1994 Peuquet propôs o modelo TRIAD, que utiliza três modelos de representação (localização, objeto e tempo) para tratar de forma distinta espaço e tempo.

- Em 1995 Raper e Livingstone propuseram o "Modelo de Dados Geomorfológicos Orientado a Objeto" (Object-Oriented geomorphologia data model - Oogeomorph), que atribui referência espaço-temporal a todas as instâncias de todas as variáveis, evitando assim o uso de uma única linha do tempo. Peuquet e Duan apresentaram o modelo "Dados Espaço-Temporais com base em Eventos" (Event-based Spatio-Temporal Data Model - ESTDM), que tem como base eventos e processos.

- Em 1999 foram propostos dois modelos que tem como objetivo representar objetos cuja geometria muda continuamente com o tempo: o modelo Linha de Vida Geoespacial (Geospatial Lifeline) (MARK et al., 1999) e o modelo Objeto em Movimento (Moving Object) (ERWIG et al., 1999). Yuan propôs um modelo de três domínios (tempo, espaço e semântica) estendendo o TRIAD proposto por Peuquet em 1994.

- Em 2004 Worboys e Hornsby apresentaram o modelo Evento Geoespacial (Geospatial Event Model - GEM), que estende os modelos geoespaciais baseados em objeto tradicionais. Este modelo adota uma abordagem híbrida permitindo três categorias de entidades: objetos, eventos e cenários. Sengupta e Yan propuseram o Modelo e Estrutura de Dados EspaçoTemporal Híbrido (Hybrid Spatio-Temporal Data Model and Structure (HST-DMS)), que tem como base eventos e processos.

Geografia Ensino \& Pesquisa, v. 16, n.1, p. 155 172, jan.jjun. 2012

Schafer, A. G.; Nogueira, R. E.

ISSN 2236- 4994 167 
- Em 2008 Choi et al. propuseram o Modelo Temporal com Base em Entidade (FeatureBased Temporal Model - FBTM) para gerenciamento da história de entidades. Este modelo adota e estende os conceitos chave do modelo objeto espaço-temporal e do modelo três domínios. Neste mesmo ano, a ISO apresentou o modelo Entidades em Movimento (Moving Feature) para representar objetos cuja geometria muda continuamente com o tempo.

\section{Considerações finais}

A tecnologia de Sistemas de Informação Geográfica percorreu um longo caminho desde as suas primeiras aplicações. Com o rápido desenvolvimento técnico e a sofisticação conceitual, cresceram as demandas e expectativas da comunidade de usuários da tecnologia.

Em aplicações avançadas, é necessária uma integração consistente do espaço e do tempo. Entretanto, como argumenta Yuan (2010), a incorporação da componente temporal em uma representação espacial não é uma tarefa trivial, pois tempo e espaço possuem propriedades distintas. Nesse contexto, o desenvolvimento de SIG com características temporais requer 0 conhecimento de algumas questões básicas, que foram abordadas no presente estudo: a) a representação de dados espaciais, temporais e espaço-temporais em SIG; b) as estruturas temporais e tipos de tempo em bancos de dados; c) a semântica dos dados espaço-temporais; d) as funções de um SIG com características espaço-temporais; e) o suporte a consultas e; f) os modelos desenvolvidos para a representação de dados espaço-temporais em SIG.

Embora questões ontológicas relacionadas especificamente à questão temporal pareçam ser conhecidas e estar razoavelmente bem entendidas para propósitos de representação em banco de dados, a natureza de conceitos que são tanto espaciais quanto temporais (por exemplo, movimento) e a interação das dimensões espacial e temporal, precisam ser melhor entendidas.

A característica estática inerente aos mapas é uma das principais razões pela qual a integração da perspectiva temporal em SIG (e a representação de fenômenos dinâmicos e entidades que se modificam) continua a ser difícil, pois a principal abstração utilizada em SIG é o mapa. A maioria das abordagens voltadas à representação de mudanças dentro dessa visão consiste na produção de uma seqüência de mapas, correspondentes a diferentes pontos em um determinado intervalo de tempo. A seqüência resultante pode ser representada em SIG como um conjunto ordenado de mapas independentes ou como uma composição de camadas (layers) espaço-temporais.

Embora sejam suficientes para muitos propósitos, essas abordagens são insuficientes para representar a informação temporal, pois somente cenários ou mudanças são representados. Segundo Couclelis (2005), esse tipo de abordagem dificulta a representação da continuidade dos fenômenos geográficos, podendo ocasionar perda de informações temporais que indiquem relações causais entre eventos, pois não registra o que pode ter ocorrido entre dois instantes no tempo.

Noções avançadas de tempo definido através de eventos, mudanças, movimento e

Geografia Ensino \& Pesquisa, v. 16, n.1, p. 155 172, jan.jjun. 2012

Inclusão da variável tempo em sistemas de informação geográfica processos vêm sendo propostas, embora muitas vezes permaneçam no nível conceitual. Embora um grande progresso no desenvolvimento de modelos de dados para SIG que vão além da abordagem retratos seqüenciais, a criação de um SIG verdadeiramente espaço-temporal permanece como um desafio não alcançado. 
Worboys (2005) argumenta que o desenvolvimento de SIGs espaço-temporais ainda tem um longo caminho a ser trilhado, e que a próxima etapa na modelagem computacional de fenômenos geográficos será a mudança de uma visão orientada a objetos para uma visão orientada a eventos. Uma abordagem completamente orientada a eventos deverá permitir uma evolução de simples consultas relacionadas a um instante no tempo (por exemplo, "o que aconteceu neste local neste momento?") para uma linguagem muito mais rica envolvendo a interação entre objetos e eventos e relacionamentos entre eventos.

\section{Referências bibliográficas}

ABRAHAM, T.; RODDICK, J. F. Survey of spatio-temporal databases. Geolnformatica, vol.3, n. 1, 1999. 61-99.

AHOLA, T.; VIRRANTAUS, K.; KRISP, J. M.; HUNTER, G. J. A Spatio-temporal population model to support risk assessment and damage analysis for decision-making. International journal of geographical information science,vol 21, n. 8, 2007. 935-953.

ARMSTRONG, M. P. Temporaliy in spatial databases. GIS/LIS'88, 1988. 880-889.

BERRY, B. J. L. Approaches to regional analysis: A synthesis. Proceedings of The association of american geographers. 1964. p. 2-11.

CAMOSSI, E.; BERTOLOTTO, M.; BERTINO, E. A multigranular object-oriented framewok supporting spatio-temporal granularity conversions. International journal of geographical information science, vol. 20, n. 8, 2006. 511-534.

CHOI, J.; SCONG, C. J.; KIM, B.; USERY, E. L. Innovations in individual feature history managementThe significance of feature-based temporal model. Geoinformatica, vol. 12, n. 1, 2008. 1-20.

COUCLELIS, H. Space, time, geography. In: LONGLEY, P. A.; GOODCHILD, M. F.; MAGUIRE, D. J.; RHIND, D. W. Geographical informations systems - Principles, techniques, management, and applications. New Jersey: Sons, John Wiley \& Sons, 2005.

COVA, T. J.; GOOGCHILD, M. F. Extending geographical representation to include fields of spacial objects. International journal of geographical information science, vol. 16, n. 6, 2002. 509-532.

DIAS, T. D. L.; DAVIS JR., C. A.; CAMARA, G. Modelos espaço-temporais. In: M. CASANOVA, G. CAMARA, C. DAVIS JR, L. VINHAS, \& G. R. DE QUEIROZ (Eds.). Curitiba: Mundogeo, 2005

EDELWEISS, N.; OLIVEIRA, J. P. Modelagem de aspectos temporais de sistemas de informação. Recife: UFPE-DI, 1994.

ERWIG, M.; TING, R. H. G.; SCHNEIDER, M.; VAZIRGIANNIS, M. Spatio-temporal data types: An approach to modeling and querying moving objects in databases. Kluwer academic publisher, 1999. 269-296.

GOODCHILD, M. F. Geographic information systems. In: Encyclopedia of social measurement..1 ed., Academic Press, 2004. p. 107-113.

GOODCHILD, M. F. Combining space and time: New potential for temporal GIS. In: KNOWLES, A. K. Placing history: How maps, spatial data and GIS are changing historical scholar ship. Redlands, $\mathrm{CA}$ : ESCRIPESS, 2008. p. 179-198.

GOODCHILD, M. F.; YUAN, M.; COVA, T. J. Towards a general theory of geographic representation in GIS. International journal of geographical information science, vol. 21, n. 3, 2007. 239-260.

Geografia Ensino \& Pesquisa, v. 16, n.1, p. 155 172, jan.jun. 2012

Schafer, A. G.; Nogueira, R. E.

ISSN 2236- 4994 
ISO, International Standard Organizations Geograpic information - Schema for moving features (ISO 19141), 2008.

HORNSBY, K.; EGENHOFER, M. J. Identity-based change: A foundation for spatio-temporal knowledge representation. International jornal of geographical information science, vol. 64, n. 1, 2000.

KJENSTAD, K. On the integration of object-based models and field-based models in GIS. Internacional journal of geographical information science, vol. 20, n. 5, May 2006. 491-509.

LANGRAN, G. Time in geographic information systems: TAYLOR \& FRANCIS, 1992.

LANGRAN, G.; CHRISMAN, N. R. A framework fonr temporal geographic information. Cartographica, Vol. 25, n. 3. 1988. 1-14.

LE, Y. Representing time in base geographic data. Dissertation (Doctor of Philosophy). University of Georgia Athens, Georgia, 2005.

LOCH, R. E. N. Cartografia: Representação, comunicação e visualização de dados espaciais. Florianópolis: editora da UFSC, 2006.

MCMASTER, R. B.; USERY, E. I. A research agenda for geographic information science. 1 ed., CRC Press, 2004.

PEERBOCUS, M. A.; MEDEIROS, C. B.; JOMIER, G.; VOISARD, A. A system for change documentation based on a spatiotemporal database. Geolnformatica, Vol. 8, n. 2, 2004. 173-204.

PEUQUET, D. J. It's about time: A conceptual framework for the representation of temporal dynamics in Geographic Information Systems. Proceedings of association of american geographers. 1994. p. 441-461.

PEUQUET, D. J. Making Space for Time: Issues in Space-Time Data Representation. Geolnformatica, Vol. 5, n. 1, 2001.11-32.

PEUQUET, D. J. Representations of space and time. New York: Guilford, 2002.

PEUQUET, D. J. Time in GIS and geographical databases. In: LONGLEY, P. A.; GOODCHILD, M. F.; MAGUIRE, D. J.; RHIND, D. W. Geographical information systems - Principles, techniques, management, and applications. New Jersey: John Wiley \& Sons, 2005. Cap. 8.

PEUQUET, D. J.; DUAN, N. An event-based spatiotemporal data model (ESTDM) for temporal analysis of geographical data. International journal of geographical information science, Vol. 9, n. 1, 1995. 7-24.

PEUQUET, D. L. A conceptual fremework and comparison of spatial data models. Cartographica, Vol. 21, n. 4, 1984. 66-113.

PRICE, R.; SRINIVASAN, B.; RAMAMOHANARAO, K. Extending the unified modeling language to support spatiotemporal applications. Proceedings of Asia technology of object oriented languages and systems. 1999. p. 163-174.

RAPER, J.; LIVINGSTONE, D. Spatio-temporal interpolation in four dimensional coastal process

models. http://www.ncgia.ucsb.edu/conf/SANTAFECDROM/sfpapers/RAPERjonathan/RAPERliv.html. 1995.

SENGUPTA, R.; YAN, C. A hybrid spatio-temporal data model and structure (HST-DMS) for efficient storage and retrieval of land use information. Transactions in GIS, Vol. 8, n. 4, 2004. 351-366.

SINTON, D. The inherent structure of information as a constraint to analysis - mapped thematic data as a case study. Harvard Papers on GIS, 7, 1978.

Geografia Ensino \& Pesquisa, v. 16, n.1, p. 155 172, jan.jun. 2012

Inclusão da variável tempo em sistemas de informação geográfica
SNODGRASS, R. T. Temporal databases. Theories and Methods of Spatio-Temporal Reasoning in Geographic Space, Berlin, 1992. 22-64.

SONDHEIM, M.; FRIESEN, P.; LUTZ, D.; MURRAY, D. Spatial archive and interchange format (SAIF) Canada. In: MOELLERING, H.; HOGAN, R. Spatial database transfer standards 2: characteristics for assessing standards and full descriptions of the national and international standards in the world (kindle edition). 1997. 
VASILIEV, I. R. Mapping time. Cartographica, Toronto, 1997. Vol. 34, n.2. 1-51.

WORBOYS, M. F. A unfied model for spatial and temporal information. The computer journal, Vol. 37, n. 1, 1994. 26-34.

WORBOYS, M. F. GIS: A computing perspective. London: Taylor and Francis, 1995.

WORBOYS, M. F. Modelling changes and events in dynamic spatial systems with reference to socio-economic units. In: FRANK, A. U.; RAPER, J.; CHEYLAN, J. P. Life and motion of socio-economic units. ESF GISDATA series. ed. Taylor and Francis, v. 8, 2001. p. 129-138.

WORBOYS, M. F. Relational databases and beyond. In: LONGLEY, P. A.; GOODCHILD, M. F.; MAGUIRE, D. J.; RHIND, D. W. Geographical information systems - Principles, techiques, management and applications. New Jersey: John Wiley \& Sons, 2005. p. 163-174.

WORBOYS, M. F.; DUNCKHAM, M. GIS: a computing perspecitve. 2a ed. EUA: CRC Press, 2004.

WORBOYS, M. F.; HEARNSHAW, H. M.; MAGUIRE, D. J. Object-oriented data modeling for spatial database. International journal of geographical information systems, Vol.4, n. 4, 1990. 369-383.

WORBOYS, M. F.; HORNSBY, K. From objects to events: GEM, the geospatial event model. Third international conference on GIScience, Springer-Verlag, Vol. 3234, 2004. 327-343.

YUAN, M. GIS and spatio-temporal modeling. Proceedings Third Internacional conference on integrating GIS and Environmental Modeling. Santa Fé, New México, USA: NGCIA. 1996.

YUAN, M. Modeling semantical, temporal and spatial information systems. In: CRAGLIA, M.; COUCLELIS, H. Geographic Information Research: Bridging The Atrantic. London: Taylor and Francis, 1996a.

YUAN, M. Use of a three-domain representation to enhance GIS support for complex spation-temporal queries. Transactions in GIS, Vol. 3, n. 2, 1999. 137-159

YUAN, M. Temporal GIS and Applications. In: SHEKHAR, S.; XIONG, H. Encyclopedia of geographic information science. Berlin: Springer-Verlag, 2008.

YUAN, M.; MARK, D. M.; EGENHOFER, M.; PEUQUET, D. Extensions to Geographic Representation. In: MCMASTER, R.; USERY, L. A research agenda for geographic information science. Boca Raton, Florida: CRC Press, 2004. cap. 5. p. 129-156.

YUAN, M.; MCINTOSH, J. A typoloy of spatiotemporal information queries. In: YUAN, M.; MCINTOSH, J. Mining spatiotemporal information systems. SHAW K.; LADNER, R.; ABDELGUERFI (eds.) Kuwer academic publisher. p. 68-82. 2002.

YUAN, M. Geographic Data Structures. In: Manual of geospatial science and technology. BOSSLER, J. (editor). New York: CRC Press, 2010.

Alexandro Gularte Schafer - Rua Prospero Ambrósio Pesce, 20. Bairro Jardim do Castelo. Bagé-RS.

E-mail: alschafer@gmail.com

Recebido em 01 de janeiro de 2011.

Aceito em 18 de março de 2012.

Geografia Ensino \& Pesquisa, v. 16, n.1, p. 155 172, jan.jun. 2012

Schafer, A. G.; Nogueira, R. E.

ISSN 2236- 4994 
Geografia Ensino \& Pesquisa, v. 16, n.1, p. 155 172, jan./jun. 2012

Inclusão da variável tempo em sistemas de informação geográfica
172
ISSN 2236-4994 